\title{
Magnon excitations and quantum critical behavior of the ferromagnet $\mathrm{U}_{4} \mathrm{Ru}_{7} \mathrm{Ge}_{6}$
}

\author{
M. P. Nascimento ${ }^{1}$, M. A. Continentino ${ }^{1}$, A. López ${ }^{2}$, Ana de Leo ${ }^{2}$, D. C. Freitas ${ }^{3}$, J. Larrea \\ J. $^{4}$, Carsten Enderlein ${ }^{5}$, J. F. Oliveira ${ }^{1}$, E. Baggio-Saitovitch ${ }^{1}$, Jirí Pospísil ${ }^{6}$, and M. B. Fontes ${ }^{1}$ \\ ${ }^{1}$ Centro Brasileiro de Pesquisas Físicas, 22290-180, Rio de Janeiro , Brazil \\ ${ }^{2}$ Departamento de Física Teórica, Universidade do Estado do Rio de Janeiro, 20550-013, Rio de Janeiro, RJ, Brazil. \\ ${ }^{3}$ Instituto de Física, Universidade Federal Fluminense, 24210-346, Niterói, RJ, Brazil \\ ${ }^{4}$ Instituto de Física, Universidade de São Paulo, 05508-090, São Paulo, SP, Brazil. \\ ${ }^{5}$ Universidade Federal do Rio de Janeiro, Rio de Janeiro-RJ, Brazil and \\ ${ }^{6}$ Faculty of Mathematics and Physics, Charles University, \\ DCMP, Ke Karlovu 5, CZ-12116 Praha 2, Czech Republic
}

(Dated: April 10, 2018)

\begin{abstract}
We present an extensive study of the ferromagnetic heavy fermion compound $\mathrm{U}_{4} \mathrm{Ru}_{7} \mathrm{Ge}_{6}$. Measurements of electrical resistivity, specific heat and magnetic properties show that $\mathrm{U}_{4} \mathrm{Ru}_{7} \mathrm{Ge}_{6}$ orders ferromagnetically at ambient pressure with a Curie temperature $T_{C}=6.8 \pm 0.3 \mathrm{~K}$. The low temperature magnetic behavior of this soft ferromagnet is dominated by the excitation of gapless spin-wave modes. Our results on the transport properties of $\mathrm{U}_{4} \mathrm{Ru}_{7} \mathrm{Ge}_{6}$ under pressures up to $2.49 \mathrm{GPa}$ suggest that $\mathrm{U}_{4} \mathrm{Ru}_{7} \mathrm{Ge}_{6}$ has a putative ferromagnetic quantum critical point (QCP) at $P_{c} \approx 1.7 \pm 0.02$ $\mathrm{GPa}$. In the ordered phase, ferromagnetic magnons scatter the conduction electrons and give rise to a well defined power law temperature dependence in the resistivity. The coefficient of this term is related to the spin-wave stiffness and measurements of the very low temperature resistivity allow to accompany the behavior of this quantity as the the ferromagnetic QCP is approached. We find that the spin-wave stiffness decreases with increasing pressure implying that the transition to the non-magnetic Fermi liquid state is driven by the softening of the magnons. The observed quantum critical behavior of the magnetic stiffness is consistent with the influence of disorder in our system. At quantum criticality $\left(P=P_{c} \approx 1.7 \pm 0.02 \mathrm{GPa}\right)$, the resistivity shows the behavior expected for an itinerant metallic system near a ferromagnetic QCP.
\end{abstract}

\section{INTRODUCTION}

The problems related to strongly correlated electronic systems are of great current interest due to the novel states of matter that can arise in these systems [1, 2]. These include their exotic magnetic properties, superconducting behavior and their phase diagrams, which exhibit quantum critical points (QCPs) [3]. QCPs are experimentally explored by doping, applied pressure or magnetic field [1]. In the case of actinide materials, the interesting properties arise from partially filled $f$-orbitals that strongly hybridize with the conduction electrons. This, together with the strong correlations among the $f$-states give rise to a variety of ground states.

The ternary compound $\mathrm{U}_{4} \mathrm{Ru}_{7} \mathrm{Ge}_{6}$ is a system with interesting magnetic properties. It has a centered body (bcc) crystalline structure of the type $\mathrm{U}_{4} \mathrm{Re}_{7} \mathrm{Si}_{6}$ [4]. The lattice parameter is $a=8.287 \AA$, and the interatomic space between the Uranium is $d_{U-U}=5.864 \AA[5]$, much larger than the Hill boundary for Uranium: $d_{U-U}=3.4$ - 3.6 A, which sets conditions for a magnetic ground state [6]. The compound $\mathrm{U}_{4} \mathrm{Ru}_{7} \mathrm{Ge}_{6}$ has the properties of a heavy fermion system, with a Kondo resistivity and a large linear term in the low temperature specific heat [5]. It orders ferromagnetically at low temperatures due to the small volume of its unit cell, which favors the RKKY interaction [5, 7, 8]. Its ferromagnetism is characterized as itinerant, although Mentink et al. [5] propose localized ferromagnetism, contrary to other works in the literature 9 11]. Under applied pressure, transport measurements show no evidence of discontinuous behavior as the ferromagnetic phase is suppressed and a non-magnetic Fermi liquid state is attained [10].

In this work, we present an extensive study of the magnetic, thermodynamic and transport properties of $\mathrm{U}_{4} \mathrm{Ru}_{7} \mathrm{Ge}_{6}$ [11] under applied magnetic field and of the latter under high applied pressures. We show that this system below its ambient pressure ferromagnetic transition at $T_{C}=6.8 \pm 0.3 \mathrm{~K}$ has its low temperature properties dominated by the presence of low energy spin-wave excitations. In systems with strong magnetocrystalline anisotropy as compounds containing $f$-states, these modes are generally quenched at low temperatures by the existence of a gap in the spin-wave spectrum due to this anisotropy. However, $\mathrm{U}_{4} \mathrm{Ru}_{7} \mathrm{Ge}_{6}$ is a unique system among the actinide materials with a negligible magneto-crystalline anisotropy [11]. This allows for the excitation of magnons at very low temperatures and these modes end up having a prominent role on its low temperature physical properties, as we show here. In particular magnons scatter the conduction electrons and have a definite importance in the electrical resistivity of ferromagnetic metals. As we apply pressure on $\mathrm{U}_{4} \mathrm{Ru}_{7} \mathrm{Ge}_{6}$ and measure its resistivity, we have a rare opportunity to observe the evolution of the spin-wave stiffness of a ferromagnetic system as it approaches the QCP. Our transport measurements show a clear softening of the magnon modes as $\mathrm{U}_{4} \mathrm{Ru}_{7} \mathrm{Ge}_{6}$ is driven to the putative ferromagnetic QCP (FQCP) with increasing pressure. In the present study, we obtain the quantum critical behavior of the stiffness of these excitations. 


\section{EXPERIMENTAL}

The sample was prepared by arc melting of its high purity metallic constituents in the ratio $\mathrm{U}: \mathrm{Ru}: \mathrm{Ge}=1: 2: 2$, under argon atmosphere, without further heat treatment, as to form the compound $\mathrm{URu}_{2} \mathrm{Ge}_{2}$. However, the X-ray diffractogram at room temperature showed a composition of $\mathrm{U}_{4} \mathrm{Ru}_{7} \mathrm{Ge}_{6}$, with additional spurious phases, as discussed in detail below. The X-ray powder diffraction (XRD) at room temperature was performed using the Bruker AXS D8 Advance II diffractometer, with LynxEye detector, $\mathrm{Cu}$ source with $\mathrm{K}_{\alpha}$ radiation.

The diffraction pattern was collected in a BraggBrentano configuration covering the angular range of 10 to 90 degrees, each step incremented by 0.02 degree. The XRD data were refined using the Rietveld method [17], implemented in the program FullProf [18], available at the Institut Laue-Langevin (ILL) website [19]. The profile function used to adjust the shape of the diffraction peaks was the pseudo-Voigt function.

Pressure-dependent resistivity measurements were carried out in a temperature range from $0.1 \mathrm{~K}$ to $10 \mathrm{~K}$ in a non-commercial Adiabatic Demagnetization Refrigerator (ADR). A standard 2.5 GPa piston cylinder type of cell was used, with a mixture of fluorinert FC70-FC77 as pressure medium, pure lead as pressure sensor at low temperatures and manganin as a manometer for loading the cell at ambient temperature. We further performed measurements of electrical resistivity under magnetic fields up to $9 \mathrm{~T}$ and respective magnetoresistance measurements in the temperature range from $1.8 \mathrm{~K}$ to $30 \mathrm{~K}$ in a commercial PPMS Dynacool from Quantum Design, at ambient pressure.

The specific heat measurements as a function of temperature were also performed in the PPMS DynaCool under different magnetic field values ranging from 0 to 7 $\mathrm{T}$, in the temperature interval from $2 \mathrm{~K}$ to $15 \mathrm{~K}$.

The magnetic characterization involved the application of external magnetic fields in DC and AC modes. The DC magnetization measurements were in field cooling (FC) mode in a field of $10 \mathrm{mT}$. For the AC susceptibility measurement, the parameters used were $H_{A C}=1 \mathrm{mT}$ and $H_{D C}=50 \mathrm{mT}$ at $3 \mathrm{kHz}$. Both type of measurements were performed from $2 \mathrm{~K}$ to $300 \mathrm{~K}$ in PPMS DynaCool, Quantum Design.

\section{SAMPLE ANALYSIS}

The XRD showed that the sample produced has a main phase of cubic crystalline structure of $\mathrm{U}_{4} \mathrm{Ru}_{7} \mathrm{Ge}_{6}$ and secondary phases. After a detailed analysis of the diffraction pattern and identification of all peaks of minor intensities, it was found that the secondary phases could probably be $\mathrm{Ru}_{2} \mathrm{Ge}_{3}$ and $\gamma$-U. The Rietveld refinement of the X-ray diffractogram was performed using first the $\mathrm{U}_{4} \mathrm{Ru}_{7} \mathrm{Ge}_{6}$ main compound phase. In the sequence, the CIF data of the other phases were added in the base of the program; after adjustment, the presence of $\mathrm{Ru}_{2} \mathrm{Ge}_{3}$ and $\gamma$-U compounds was confirmed as secondary phases in the sample. The CIF data were obtained from the Inorganic Crystal Structure Database (ICSD).

Fig. 11 shows the refinement of the XRD, where the black circles are the experimentally observed data, while the solid red line is the standard calculated by the refinement. The allowed Bragg positions are represented in vertical green bars, where each level corresponds to the peaks of the Bragg planes of each of the phases found. The planes (hkl) shown in the figure correspond to the peaks of the diffractogram of the predominant phase $\mathrm{U}_{4} \mathrm{Ru}_{7} \mathrm{Ge}_{6}$, which appears in the amount of $78.08 \%$. The parameters of the crystalline lattice and the amounts of each of the phases found as results of the refinement, as well as their quality $\mathrm{R}$ factors, are described in Table 1. The crystallographic data-sheet ICSD 192067 [9] was used for the refinement of the phase $\mathrm{U}_{4} \mathrm{Ru}_{7} \mathrm{Ge}_{6}$. In Table 1 , it is seen that the lattice parameters of all phases are in agreement with the literature [5, 7, 21, 22]. The interatomic distance of the Uranium of the major phase $\mathrm{U}_{4} \mathrm{Ru}_{7} \mathrm{Ge}_{6}$ is $\mathrm{d}_{U-U}=5.866 \AA$, also agreeing with the literature [5, 7].

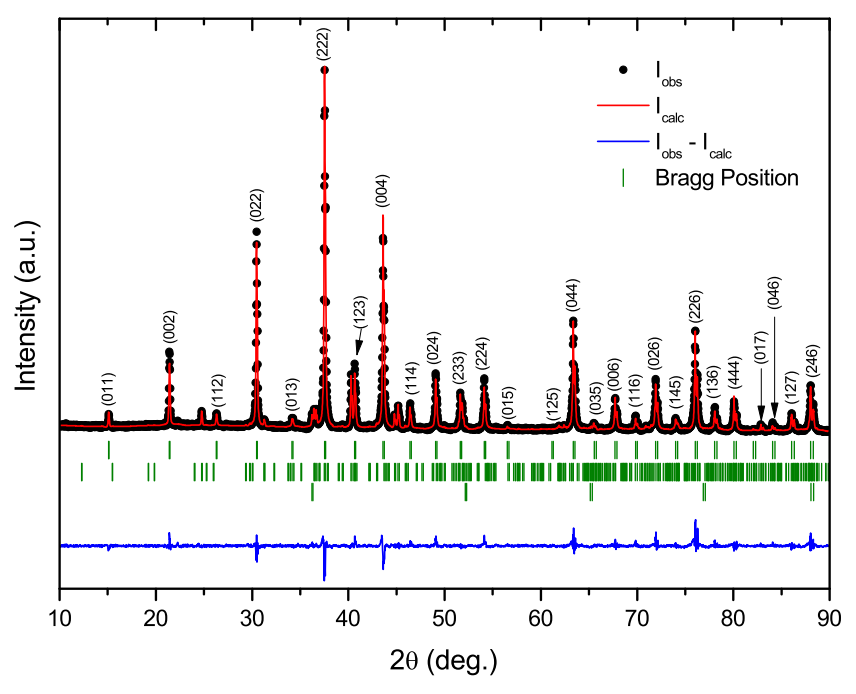

FIG. 1. (Color online) Result of the Rietveld refinement of the X-ray diffraction pattern of the sample. The experimentally observed data are the black circles and the calculated standard is the solid red line. The allowed Bragg positions are represented in vertical green bars, where each level corresponds to each of the phases found: $\mathrm{U}_{4} \mathrm{Ru}_{7} \mathrm{Ge}_{6}(78.06 \%)$, $\mathrm{Ru}_{2} \mathrm{Ge}_{3}(20.78 \%)$ and $\gamma-\mathrm{U}(1.16 \%)$. The difference pattern $\left(I_{\text {obs }}-I_{\text {calc }}\right)$ is represented in solid blue line. The planes (hkl) shown are of the major phase $\mathrm{U}_{4} \mathrm{Ru}_{7} \mathrm{Ge}_{6}$.

While Uranium in its allotropic forms has a higher resistivity than copper [25], it has a weak paramagnetic behavior, exhibiting paramagnetism almost independent of temperature [26, 27]; the second spurious phase of the sample, $\mathrm{Ru}_{2} \mathrm{Ge}_{3}$, is semiconductor and strongly diamagnetic, exhibiting a paramagnetic 
contribution above $900 \mathrm{~K}$ due to the structural transition [28], from orthorhombic (center-symmetric) to tetragonal (non-symmetrical) [29]. Thus, in this work, we state that the low temperature magnetic, thermodynamic and transport properties of our sample are due to the main phase $\mathrm{U}_{4} \mathrm{Ru}_{7} \mathrm{Ge}_{6}$ with negligible contribution from the secondary phases.

TABLE I. Crystallographic parameters obtained from the Rietveld refinement for the sample.

\begin{tabular}{lccc}
\hline \hline Phase & $\mathrm{U}_{4} \mathrm{Ru}_{7} \mathrm{Ge}_{6}$ & $\mathrm{Ru}_{2} \mathrm{Ge}_{3}$ & $\gamma$-U \\
\hline \hline Composition & $78.06 \%$ & $20.78 \%$ & $1.16 \%$ \\
\hline Crystalline structure & $\mathrm{cubic}$ & orthorhombic & cubic \\
\hline Space group & $\mathrm{Im} \overline{3}$ & Pbcn & $\mathrm{Im} \overline{3} \mathrm{~m}$ \\
\hline Datasheet CIF-ICSD & 192067 & 85205 & 44392 \\
\hline Lattice parameter & & & \\
a $(\AA)$ & 8.295813 & 11.436 & 3.504737 \\
b $(\AA)$ & - & 9.238 & - \\
$\mathrm{c}(\AA)$ & - & 5.716 & - \\
\hline \hline
\end{tabular}

Quality of the refinement: $R_{p}=19.3 \% ; R_{w p}=16.9 \%$; $R_{\text {exp }}=8.98 \% ; \chi^{2}=3.537 ; S=1.881$.

${ }^{*}$ Crystalographic Information File - Inorganic Crystal Structure Database

\section{MAGNETIC PROPERTIES AND EVIDENCE FOR SPIN-WAVES}

The inverse of the magnetic AC-susceptibility $\chi_{A C}(T)$ of our sample as a function of temperature is shown in Fig. 2 from $2 \mathrm{~K}$ to $18 \mathrm{~K}$. The data shows a peak at $T_{C}=6.8 \mathrm{~K}$ that we identify as the Curie temperature, below which $\mathrm{U}_{4} \mathrm{Ru}_{7} \mathrm{Ge}_{6}$ becomes ferromagnetic. This coincides with the $T_{C}$ found in Ref. [5] $\left(T_{C}=6.8\right.$ $\mathrm{K}$ ), but is smaller than those found in Refs. [7, 10] that range in the interval between $10.0-13.0 \mathrm{~K}$. Above $T_{C}$ the susceptibility follows a Curie-Weiss law from which we extract an effective magnetic moment of $\approx 2.14 \mu_{B}$ per Uranium atom [30]. This is large compared to the value obtained from the saturation magnetization in large magnetic fields $\left(0.2 \mu_{B}\right.$ per Uranium atom) [5, 11], but smaller than the value of $2.54 \mu_{B}$ extracted from the Curie-Weiss behavior of the susceptibility at high temperatures $(300 \mathrm{~K} \lesssim \mathrm{T} \lesssim 500 \mathrm{~K})$ [7]. It is interesting that the Curie-Weiss temperature $\theta$ indicated by an arrow in Fig. 2 is very close to the ferromagnetic critical temperature $T_{C}$ obtained from the peak in $\chi_{A C}$. This mean-field behavior is consistent with the rather large Uranium moment in a cubic structure and indicates that ferromagnetic fluctuations are important only close to $T_{C}$.

In ferromagnetic systems, metallic or insulators, below $T_{C}$ the low temperature magnetic elementary excitations are long wave-length spin-waves with dispersion relation $\hbar \omega_{k}=\Delta+D k^{2}$. The gap $\Delta$ may be due to the magneto-

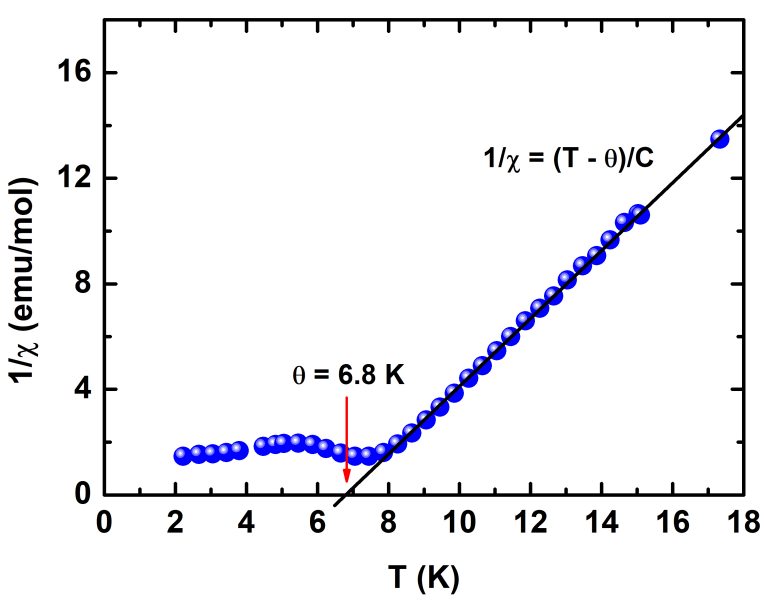

FIG. 2. (Color online) The inverse of the magnetic ACsusceptibility $\chi_{A C}(T)$ of the sample as a function of temperature for a frequency of $3 \mathrm{kHz}$, an AC-field of $1 \mathrm{mT}$ and a DC field $H_{D C}=50 \mathrm{mT}$. The peak marked with an arrow is the Curie-Weiss temperature that is very close to the ferromagnetic transition of $\mathrm{U}_{4} \mathrm{Ru}_{7} \mathrm{Ge}_{6}$, as indicated by the peak in the susceptibility.

crystalline anisotropy, dipolar interactions or to the Zeeman energy if an external magnetic field $H_{a}$ is applied in the material. The quantity $D$ is the spin-wave stiffness of the magnetic system. For a soft ferromagnet with negligible anisotropy, in zero field and in the temperature range of our experiments, the gap in general can be neglected and the spectrum is purely quadratic in the wave-vector $k$. Notice that in this case, the mode $\omega_{k=0}=0$ is the Goldstone mode of the rotational invariant system [3].

Fig. 3 shows the low temperature behavior of $C_{M} / T$, the molar specific heat divided by temperature, of $\mathrm{U}_{4} \mathrm{Ru}_{7} \mathrm{Ge}_{6}$. We took into account that only $78 \%$ of the total molecular weight is due to the main phase. The data is plotted in such a way to put in evidence a contribution proportional to $T^{3 / 2}$ associated with gapless ferromagnetic magnons with a quadratic dispersion. There is also a large linear temperature dependent term that is due to the heavy quasi-particles of the metallic $\mathrm{U}_{4} \mathrm{Ru}_{7} \mathrm{Ge}_{6}$ compound. A phonon contribution to the specific heat becomes apparent in a plot of $C_{M} / T$ versus $T^{2}$, where a linear behavior is observed in the temperature interval from $\approx 15 \mathrm{~K}$ to $30 \mathrm{~K}$. The Debye temperature obtained from the inclination of this line is $\Theta_{D}=276$ $\mathrm{K}$ [5]. The data shows that this phonon contribution can be safely neglected in the temperature region below $T_{C}$. The coefficient of the linear temperature dependent term of the specific heat obtained from the fit in Fig. 3 with $C_{M} / T=\gamma_{0}+\delta T^{3 / 2}$ is $\gamma_{0}=102 \mathrm{~mJ} / \mathrm{mol} \mathrm{U}$ $\mathrm{K}^{2}$, similar to that given in the literature for this material [5, 10, 11]. The coefficient of the spin-wave contribution obtained from this fit is $\delta=0.038 \mathrm{~J} / \mathrm{mol} \mathrm{K}^{5 / 2}$. In 
spin-wave theory, for a cubic ferromagnetic system with gapless magnon excitations, the expression for the contribution of these modes to the low temperature specific heat per unit volume is calculated as [12],

$$
C_{V}=\frac{1}{V}\left(\frac{\partial E}{\partial T}\right)_{V}=\frac{15}{4} \zeta\left(\frac{5}{2}\right)\left(\frac{1}{4 \pi D}\right)^{3 / 2} k_{B}^{5 / 2} T^{3 / 2}
$$

This expression allows to obtain the spin-wave stiffness $D$ from the coefficient of the $T^{3 / 2}$ term of the specific heat in the experimental data as shown in Fig. 3. Notice that $C_{M}=C_{V} \mathcal{V}_{M}$ with $C_{V}$ given by Eq. 1 and $\mathcal{V}_{M}$ the molar volume. We get, using the value of $\delta$ above, $D=32 \pm 1$ meV $\AA^{2}$. The error here is mainly due to uncertainty in the volume of the sample.

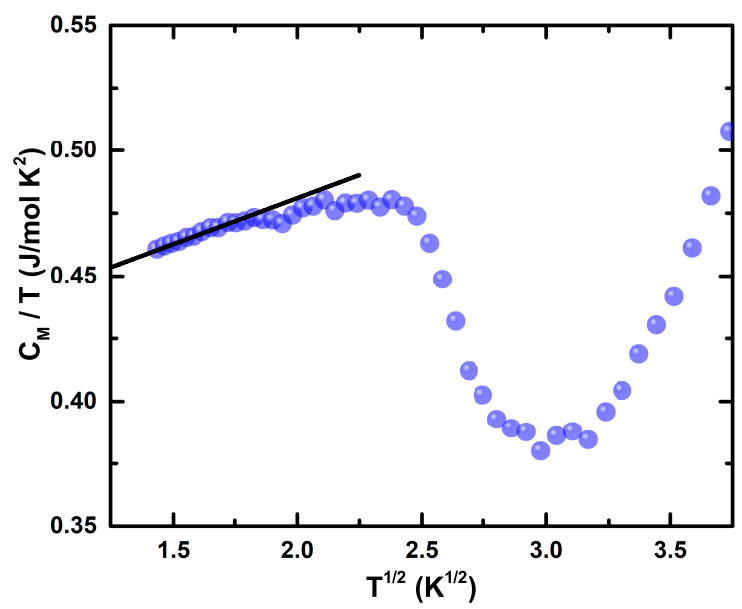

FIG. 3. (Color online) Molar specific heat plotted as $C_{M} / T$ versus $T^{1 / 2}$ to put in evidence a $T^{3 / 2}$ contribution due to ferromagnetic magnons. The extrapolation of the line gives a linear temperature contribution with a coefficient $\gamma_{0}=0.407$ $\mathrm{J} / \mathrm{mol} \mathrm{K}^{2}$. The coefficient of the $T^{3 / 2}$ term obtained from the inclination of the line turns out to be $\delta=0.038 \mathrm{~J} / \mathrm{mol} \mathrm{K}^{5 / 2}$.

Fig. 4 shows the normalized low temperature magnetization of $\mathrm{U}_{4} \mathrm{Ru}_{7} \mathrm{Ge}_{6}$ as a function of $T^{3 / 2}$. The linear behaviour of the magnetization in this plot implies that at low temperatures it decreases according to a Bloch's $T^{3 / 2}$ law. This is a clear signature that this decrease is due to the thermal excitation of ferromagnetic magnons [12]. Bloch's law yields,

$$
M(T) / M(0)=1-B T^{3 / 2}
$$

at low temperatures [12]. The coefficient $B$ is related to the spin-wave stiffness by [12],

$$
B=\frac{\zeta(3 / 2) g \mu_{B}}{M(0)}\left(\frac{k_{B}}{4 \pi D}\right)^{3 / 2} .
$$

Using the experimental value of $B$ obtained from Fig. 4 in Eq. 3. we find $D=27 \pm 1 \mathrm{meV}^{2}$, where the error comes from the uncertainty on the volume of the sample. In Eq. 2, $\mathrm{M}(0)=6.1 \times 10^{6} \mathrm{emu} / \mathrm{m}^{3}$

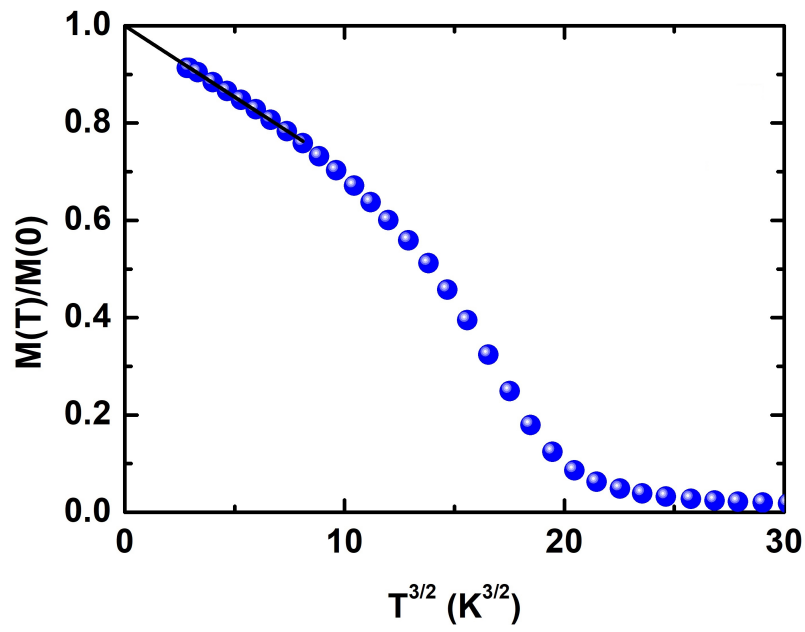

FIG. 4. (Color online) Normalized low temperature magnetization of the ferromagnet $\mathrm{U}_{4} \mathrm{Ru}_{7} \mathrm{Ge}_{6}$ versus $T^{3 / 2}$ measured in a field cooled of $10 \mathrm{mT}$. At low temperatures, the magnetization decreases with a Bloch $T^{3 / 2}$ law. We get for the coefficient in Eq. 2. $\mathrm{B}=0.030 \mathrm{~K}^{-3 / 2}$.

The values for the spin-wave stiffness obtained above from the low temperature specific heat and magnetization measurements, $D=32 \pm 1.0 \mathrm{meV} \AA^{2}$ and $D=$ $27 \pm 1.0 \mathrm{meV} \AA^{2}$ respectively, are consistent and of the expected order of magnitude for a soft metallic ferromagnet with a Curie temperature of $T_{C} \approx 10 \mathrm{~K}$. For example, in ferromagnetic $\mathrm{Ni}$ with $T_{C} \approx 631 \mathrm{~K}[12]$ the experimentally obtained spin-wave stiffness ranges from $D \approx 422 \mathrm{meV}^{2}$ to $D \approx 555 \mathrm{meV} \AA^{2}[13$, if extracted from Bloch's law or measured directly by neutron scattering, respectively. These results strongly support the idea that ferromagnetic magnons play an important role in the thermodynamic properties of the cubic ferromagnetic $\mathrm{U}_{4} \mathrm{Ru}_{7} \mathrm{Ge}_{6}$ below its Curie temperature.

\section{TRANSPORT PROPERTIES}

In ferromagnetic metals, the scattering of conduction electrons by ferromagnetic spin-waves gives rise to a $T^{2}$ temperature dependent contribution to the electrical resistivity of these materials [14]. This $T^{2}$ contribution has indeed been found in a previous study of $\mathrm{U}_{4} \mathrm{Ru}_{7} \mathrm{Ge}_{6}[10]$, but it has been attributed to electron-electron scattering as in strongly correlated paramagnetic metals. However, in a long-range ordered ferromagnetic metal, with polarized bands, and gapless spin-wave modes, the main scattering is due to these elementary excitations. In fact, this is the main form electron-electron scattering assumes in an itinerant ferromagnetic metal. Here, we give evidence that in ferromagnetic $\mathrm{U}_{4} \mathrm{Ru}_{7} \mathrm{Ge}_{6}$ substantial part of the $T^{2}$ term in its resistivity is due to electron-magnon 
scattering. This contribution to the resistivity is given by [14],

$$
\rho=A T^{2}=\frac{32}{3} \zeta(2) \pi^{2} \rho_{0}\left(\frac{\Delta E}{E_{F}}\right)\left(\frac{k_{B} T}{D k_{F}^{2}}\right)^{2}
$$

where $\rho_{0}$ is a constant with units of resistivity, $k_{F}$ and $E_{F}$, the Fermi wave-vector and Fermi energy of the $f$-electrons, respectively. The quantity $\left(\Delta E / E_{F}\right)=$ $2 q_{\max } / k_{F}$ where $q_{\max }$ is the maximum wave-vector for which the quadratic spin-wave dispersion relation, $\hbar \omega=$ $D k^{2}$, holds. The spin-wave stiffness appears in the denominator of this equation, such that, the softer the magnon modes the larger is this contribution to the resistivity. At a FQCP, where the spin-wave stiffness vanishes, the resistivity of the metal is given by [14],

$$
\rho=64 \pi \rho_{0} \Gamma\left(\frac{8}{3}\right) \zeta\left(\frac{5}{3}\right)\left(\frac{3 \pi k_{B} T}{E_{F}}\right)^{5 / 3} .
$$

In the next section, we will present results for the electric resistivity of our sample as a function of temperature for different applied pressures and magnetic fields. As pressure increases, the ferromagnetic Curie temperature vanishes smoothly at a FQCP at a critical pressure, $P_{c}=1.7 \pm 0.02 \mathrm{GPa}$. The resistivity curves we obtain present no hysteresis for any pressure. We find no evidence of a behavior that could indicate a first order transition as the Curie temperature of the sample is reduced and made to vanish. As we accompany the variation of the coefficient of $T^{2}$ term with increasing pressure, we observe a smooth increase of this coefficient that we attribute entirely, according to Eq. 4. to a decrease of the spin-wave stiffness as the FQCP is approached. At the critical pressure the resistivity follows a $T^{5 / 3}$ behavior in agreement with Eq. 5 .

\section{A. Pressure experiments}

Fig. 5 shows the low temperature behavior of the electrical resistivity of $\mathrm{U}_{4} \mathrm{Ru}_{7} \mathrm{Ge}_{6}$ as a function of pressure. All pressure experiments were carried out in zero external magnetic field. The electrical resistivity behaves smoothly, with no detectable hysteresis for all pressures of the experiments. At very low temperatures, it presents a $T^{2}$ dependence, both above and below the critical pressure $P_{c} \approx 1.7 \mathrm{GPa}$ and is well described by, $\rho=\rho_{0}+A(P) T^{2}$. The exception is for pressures very close to $P_{c}$ where $\rho(T) \propto T^{5 / 3}$, as shown in Fig. 6. This is the expected power law behavior for a ferromagnetic metallic system close to a FQCP (see Eq. 5).

In Fig. 7, we show physical parameters obtained from the resistivity data as a function of pressure.

- In principle, the observation of a clear cut-bend (or kink) in the $T$-dependence of electrical resistivity is an indication of an onset of magnetic ordering. The precise determination of $T_{C}$ is obtained from

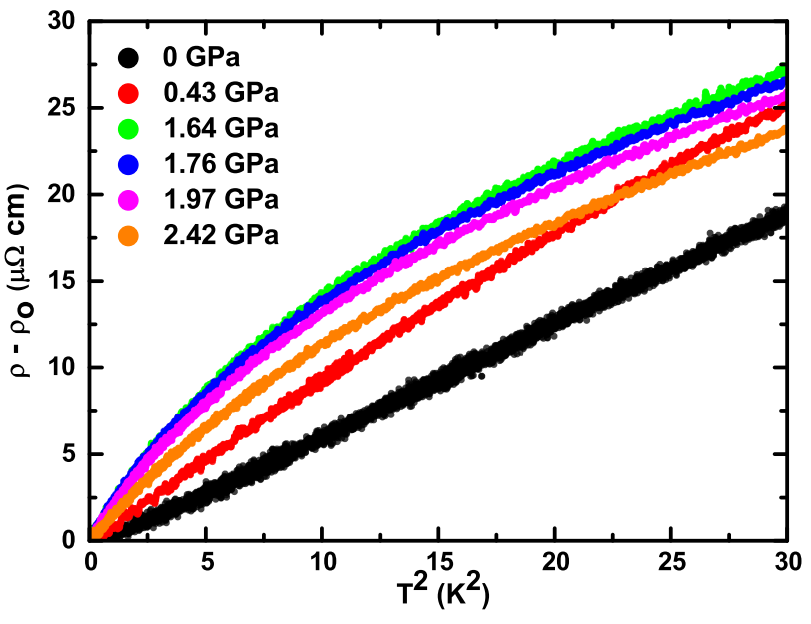

FIG. 5. (Color online) Resistivity versus temperature of the ferromagnet $\mathrm{U}_{4} \mathrm{Ru}_{7} \mathrm{Ge}_{6}$ for different applied pressures above and below the critical pressure. The residual resistivity and the coefficients of the low temperature $T^{2}$ terms for different pressures are shown in Fig. 7

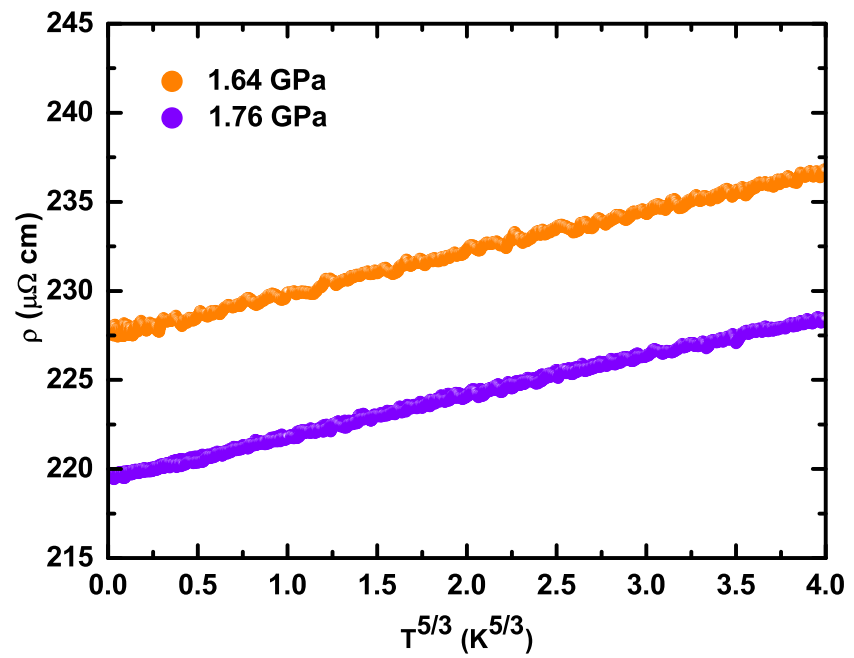

FIG. 6. (Color online) Low temperature resistivity of the ferromagnet $\mathrm{U}_{4} \mathrm{Ru}_{7} \mathrm{Ge}_{6}$ for pressures very close to the pressure where ferromagnetism is suppressed. The plot puts in evidence a $T^{5 / 3}$ power law behavior expected for an itinerant $3 d$ ferromagnet at a FQCP (see Eq. 51).

the second derivative of the smoothed electrical resistivity data [15]. In the upper panel of Fig. 7 . we have plotted the $T_{C}$ obtained in this way and draw through these points a curve from a fit using the expected power law behavior for an itinerant FQCP, $T_{C} \propto\left|P_{c}-P\right|^{\psi}$, where the shift exponent [3] $\psi=z /(d+z-2)=3 / 4$, since the dynamic exponent is $z=3$ in this case [3]. The curve gives a reasonable description of the pressure dependent Curie temperatures. 

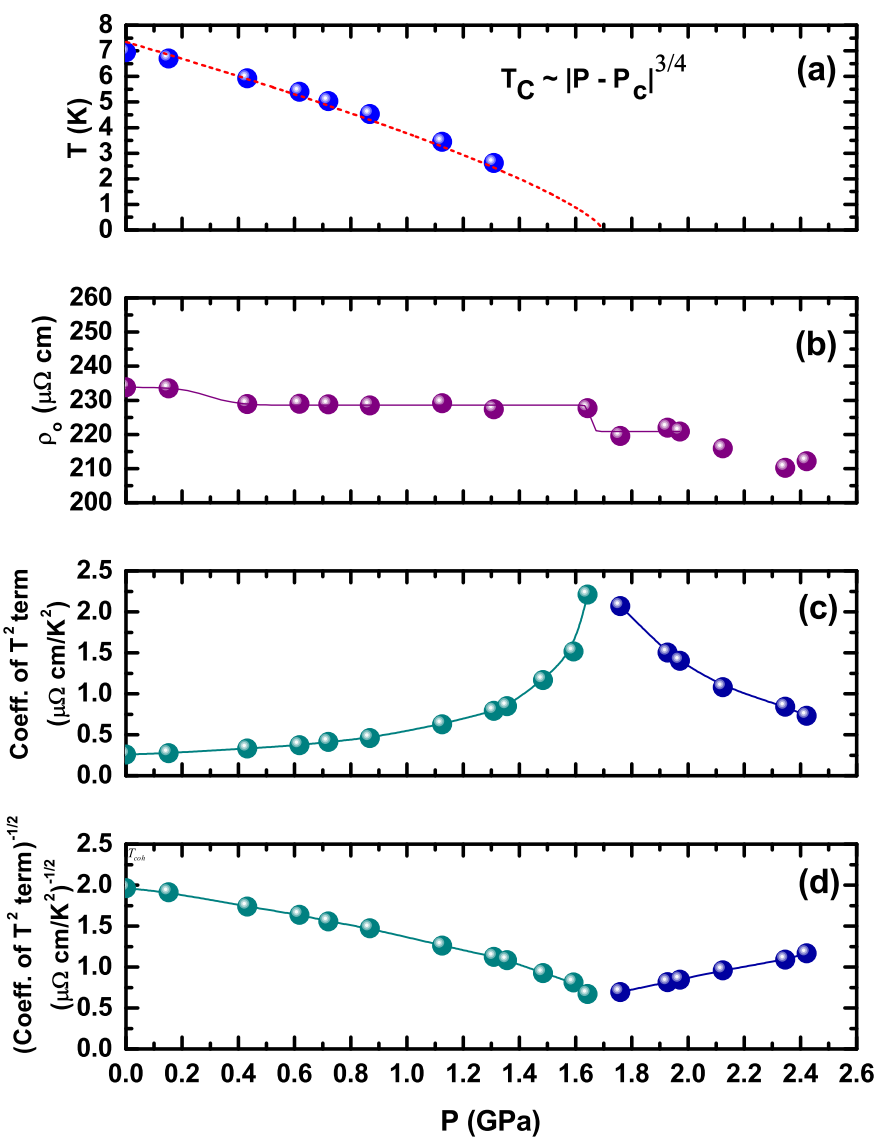

FIG. 7. (Color online) Parameters extracted from the temperature dependent resistivity curves for different pressures. Upper panel, the Curie temperature obtained from the second derivative of the resistivity (see text). The dashed line corresponds to a fitting with the expression $T_{C} \propto\left|P_{c}-P\right|^{\psi}$ with $\psi=3 / 4=0.75$, the expected shift exponent for a $3 \mathrm{~d}$ itinerant ferromagnet (see text), and $P_{c}=1.7 \mathrm{GPa}$. The panel below shows the residual resistivity as a function of pressure. The lower two panels refer to the coefficients of the $T^{2}$ term in the resistivity, above and below $P_{c}$.

- The next panel shows the pressure dependence of the residual resistivity. This is nearly constant in the ferromagnetic phase, with a small drop close to the critical pressure.

- The last two panels refer to the pressure dependence of the coefficient of the $T^{2}$ term of the resistivity. They rise on both sides of the phase diagram as the critical pressure is approached from below and above in a non-symmetric fashion. In the paramagnetic phase, above $P_{c}$, this term is due to scattering by paramagnons and its coefficient is proportional to the square of the inverse of the coherence temperature [16], $T_{c o h} \propto\left|P-P_{c}\right|^{\nu z}$, with $\nu z=3 / 2$ for a three dimensional itinerant ferromagnetic system [16]. As can be seen in the last two panels, we do not have enough data for $T_{c o h}$

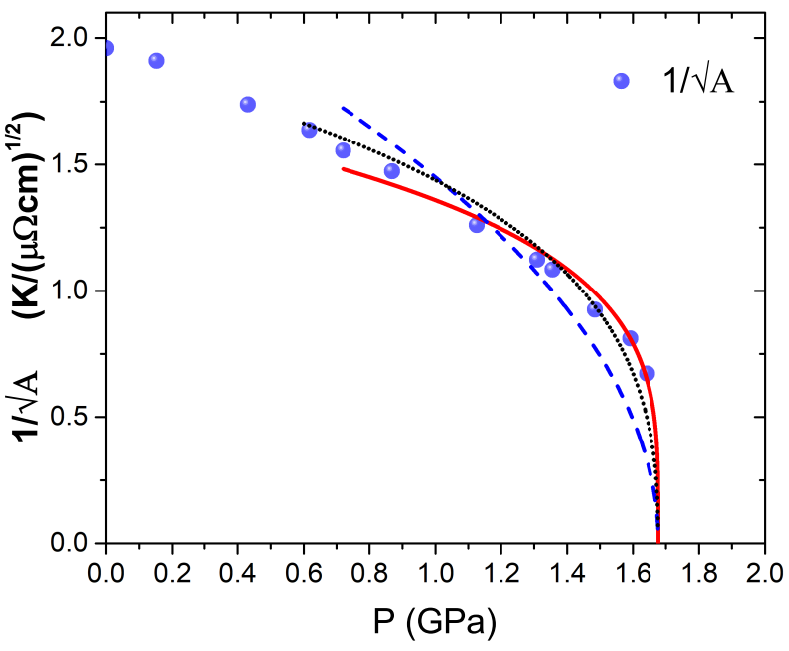

FIG. 8. (Color online) Pressure dependence of the quantity $1 / \sqrt{A}$, that mimics that of the spin-wave stiffness $D$. Dashed line is the mean-field prediction $D \propto m$, while the full and dotted lines are best fittings using Eqs. 6] and 7 respectively. In every case $P_{c}=1.64 \mathrm{GPa}$.

close to the critical pressure to be able to determine its power law dependence with the distance from criticality. Sufficiently far from $P_{c}, T_{\text {coh }}$ depends linearly on this distance, which suggests local quantum critical behavior [3].

- For pressures below $P_{c}$, in the ferromagnetic phase,

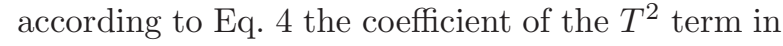
the resistivity is related to spin-wave stiffness $D$, $A(P) \propto 1 / D^{2}$. In itinerant $3 \mathrm{~d}$ ferromagnets, the coupling of the order parameter to particle-hole excitations give rise to a non-analytic behavior of the spin-wave stiffness as a function of the magnetization $m[39]$. For a disordered quantum itinerant 3d ferromagnet,

$$
D(m \rightarrow 0)=c_{3} m\left[m^{-1 / 2}+O(1)\right]
$$

while for the clean system,

$$
D(m \rightarrow 0)=\tilde{c}_{3} m[\ln (1 / m)+O(1)]
$$

where $c_{3}$ and $\tilde{c}_{3}$ are positive constants [39]. In a $3 \mathrm{~d}$ quantum metallic ferromagnet the magnetization vanishes close to the FQCP as $m \propto\left|P_{c}-P\right|^{\beta}$ with a mean-field exponent $\beta=1 / 2$. In Fig. 8 we plot the pressure dependence of the quantity $1 / \sqrt{A}$ that mimics that of the spin-wave stiffness for pressures approaching the FQCP. We compare the simple mean-field result $D \propto m \propto \sqrt{\left(P_{c}-P\right)}$ with the results of Eqs. 6 and 7 The results for the clean and disordered ferromagnet are rather similar, but clearly they give a better description of the data than the simple mean-field. 


\section{EFFECT OF AN EXTERNAL MAGNETIC FIELD}

In this section, we study the effect of an applied magnetic field on the thermodynamic and transport properties of our sample at ambient pressure. In ferromagnets, a magnetic field is the conjugate of the order parameter and destroys the thermodynamic phase transition. This is different from the antiferromagnet where a uniform magnetic field just shifts the transition. The low temperature magnetic excitations of the ferromagnet in an external magnetic field are still magnons, but they become partially quenched by a Zeeman gap due to the coupling of the magnetic moments to the field. This

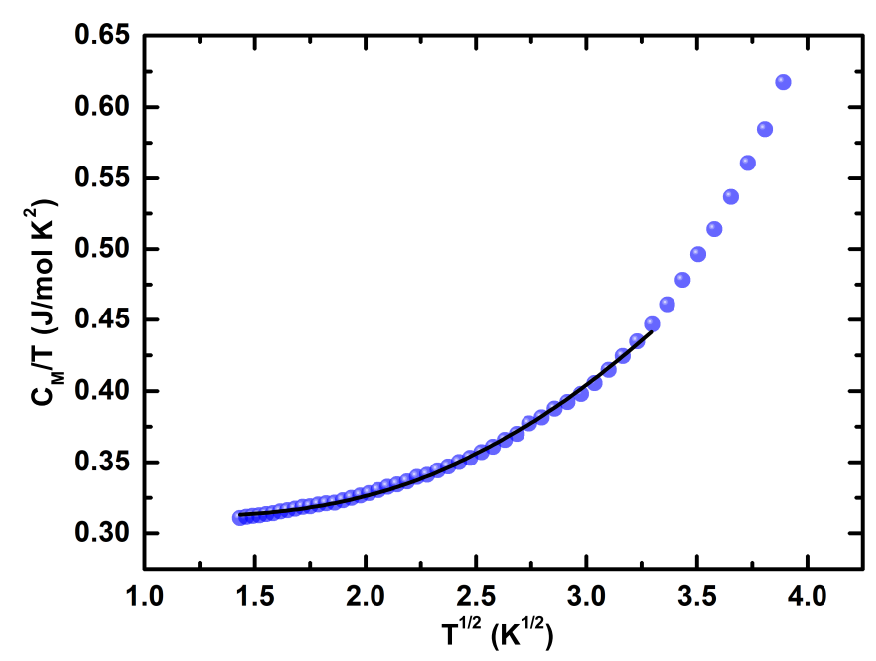

FIG. 9. (Color online) Specific heat of $\mathrm{U}_{4} \mathrm{Ru}_{7} \mathrm{Ge}_{6}$ as a function of temperature in an external magnetic field $H_{a}=7 \mathrm{~T}$. The fitting curve $C_{M} / T=\gamma+b \sqrt{T} e^{-\Delta / T}$ includes an exponential term that accounts for the freezing of the magnons by the external field. The parameters $\gamma=310 \mathrm{~mJ} / \mathrm{mol} \mathrm{K}^{2}$ and $\Delta=9.1 \mathrm{~K}$.

reduces the influence and contribution of spin-waves to the low temperature properties, i.e., for $k_{B} T<\Delta$, where $\Delta$ is the Zeeman gap.

In Fig. 9] we show the specific heat as a function of temperature in an applied field of $7 \mathrm{~T}$. The low temperature specific heat is well fitted by the expression $C_{M} / T=\gamma+b \sqrt{T} \exp (-\Delta / T)$. The exponential term takes into account the quenching of the magnons by the Zeeman gap $\Delta=\left(g \mu_{B} S H_{a}\right) / k_{B}$, expressed here in temperature scale. From this fit we can determine the coefficient of the linear term $\gamma\left(H_{a}\right)$ and the Zeeman gap $\Delta\left(H_{a}\right)$ for several values of the external magnetic field, as shown in Figs. 10] and 11] respectively. Fig. 10] shows that the coefficient of the linear term in the specific heat is reduced as the external field is applied. The simplest interpretation for this effect is that the Zeeman splitting of the polarized bands causes a decrease in the density of states at the Fermi level [40]. This behavior of $\gamma\left(H_{a}\right)$ is quite distinct from that in antiferromagnet heavy fermions [41],

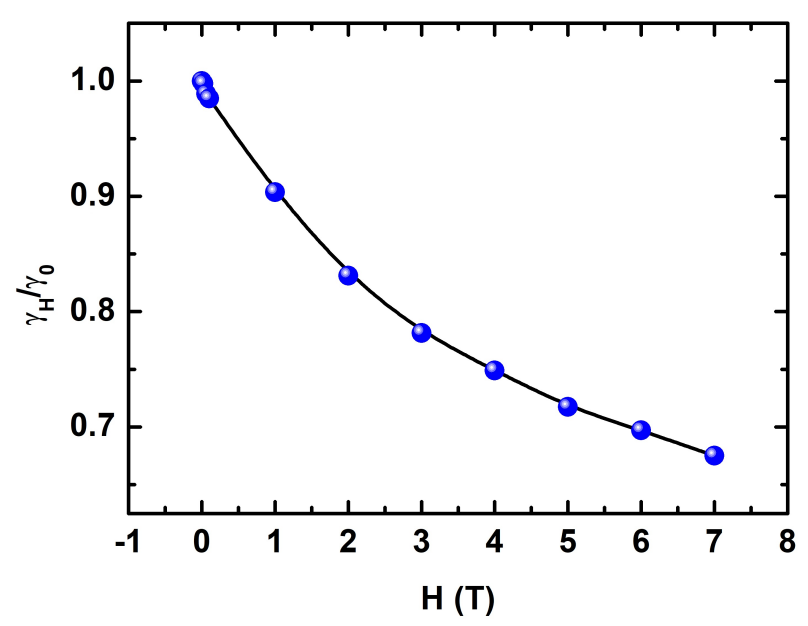

FIG. 10. (Color online) The coefficient of the linear term in the specific heat as a function of the external magnetic field. The line is a guide to the eyes.

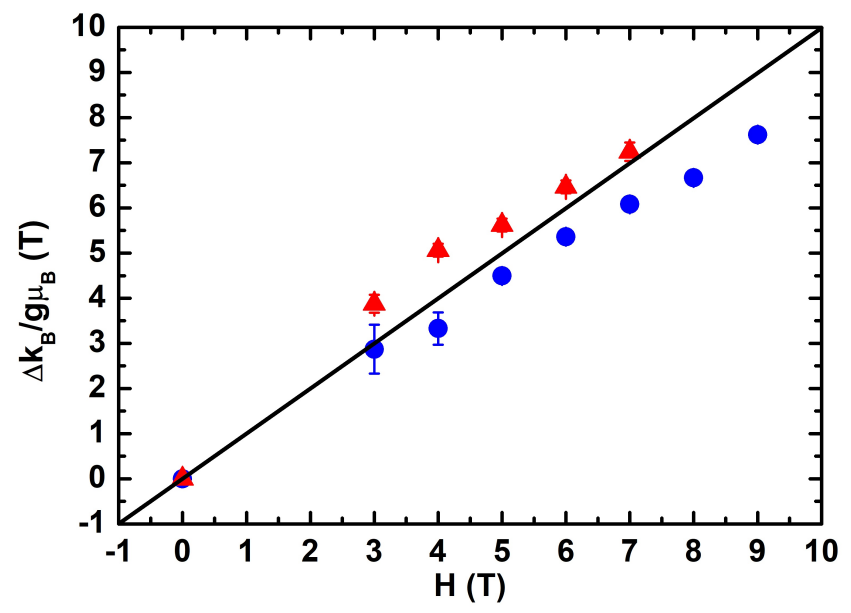

FIG. 11. (Color online) The spin-wave gaps extracted from the specific heat data (triangles) and from the resistivity (circles) using the expressions in the text. The straight line is the result expected from the simplest spin-wave theory (see text).

as expected from the different roles of $H_{a}$ in these systems.

The suppression of the magnons by the magnetic field also decreases the low temperature electrical resistivity due to a partial freezing of the electron-magnon scattering. The low temperature electrical resistivity shown in Fig. 12 for $H_{a}=7 \mathrm{~T}$ is well described by the expres- 


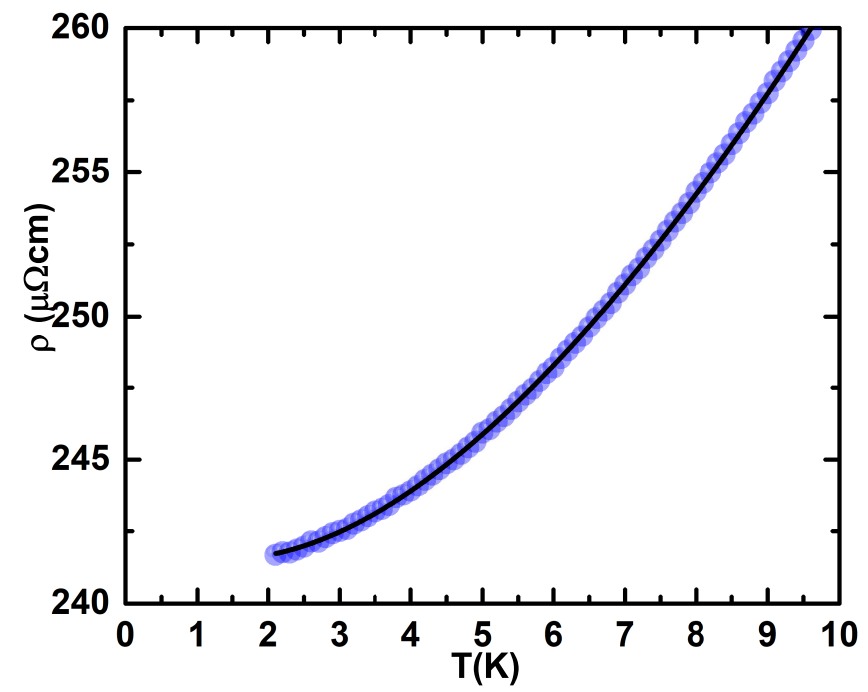

FIG. 12. (Color online) Resistivity of our sample as a function of temperature in an external magnetic field $H_{a}=7 \mathrm{~T}$. The fitting curve $\rho=\rho_{0}+b_{r} T^{2} e^{-\Delta / T}+c_{r} T e^{-\Delta / T}$ (black) takes into account the quenching of the magnons by the magnetic field that suppresses the electron-magnon scattering at very low temperatures (see text). The gap for this field is $\Delta=6.5$ K.

sion 42 ]

$$
\rho=\rho_{0}+a \Delta T e^{-\Delta / T}\left(1+2 \frac{T}{\Delta}\right) .
$$

The gap $\Delta$ (units of temperature) extracted from the resistivity data for several values of the applied magnetic field is shown in Fig. 11] The gaps obtained from the transport and thermodynamic data are in satisfactory agreement. The straight line in this plot show the expected value for the Zeeman gap in the simplest (non self-consistent) spin-wave theory [31.

For completeness, we now present magneto-resistance results at ambient pressure for our sample. Resistivity is measured as a function of magnetic field for fixed temperatures below the Curie temperature. For small fields, $H_{a} \ll 1 \mathrm{~T}$ and very low temperatures, the magnetoresistance is positive reaches a maximum at $H_{\max }$ and then decreases almost linearly with field for $H_{a}>$ $H_{\max }$. Magneto-resistance of multi-band ferromagnetic metals, like transition metals, has been intensively studied both experimentally and theoretically [43]. Raquet et al. [43] have shown that for systems with a light $c$ band of conduction electrons and a heavy $f$-band of quasiparticles, intra-band scattering in the conduction c-band can be neglected. Notice that due to the strong $c-f$ hybridization in $\mathrm{U}_{4} \mathrm{Ru}_{7} \mathrm{Ge}_{6}$ [1] the bands have a hybrid character and those referred above are in fact are mostly c-band and mostly f-band. Considering electronmagnon scattering, which involves intra-band $f-f$ and inter-band $c-f$ spin flip process, Raquet et al. [43] have shown that in the presence of a magnetic field, the magneto-resistance roughly follows a $H_{a} \ln H_{a}$ dependence for temperatures above approximately $T_{C} / 5$. In Fig. 13 we plot the magneto-resistance defined as $\Delta \rho=\rho\left(H_{a}, T\right)-\rho\left(H_{\max }, T\right)$ as a function of $H_{a}-H_{\max }$ for different fixed temperatures. $H_{\max }$ is the value of the magnetic field for which the magnetoresistance reaches a maximum before starts to decrease. We attribute the positive magneto-resistance at low fields and temperatures to the existence of domain walls that are eventually removed at $H_{\max }$. Fig. 13 also shows the fittings

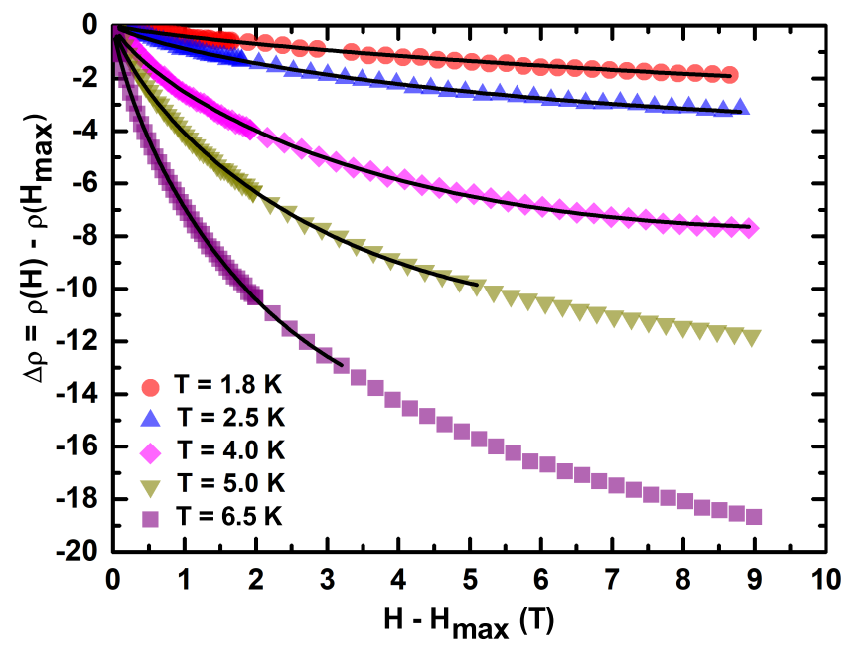

FIG. 13. (Color online) Magneto-resistance of our sample as a function of of magnetic field for several fixed temperatures. The lines are two-parameters fittings $(a$ and $b)$ using the expression $\Delta \rho=a\left(H-H_{\max }\right) \ln \left(H-H_{\max }\right) / b$ as given in the text [43].

using the simple logarithmic law obtained in Ref. [43], $\Delta \rho=a \delta H \ln (\delta H / b)$, with $\delta H=H-H_{\max }$. It is clear it gives a good description of our data.

\section{CONCLUSIONS}

The study of systems close to quantum criticality is an exciting area of research. In the case of itinerant ferromagnets driven to a magnetic instability, there are theoretical and experimental evidences that quantum critical behaviour is avoided and a first order transition occurs before the FQCP is reached [44, 45]. In this work we present a thorough investigation of the ferromagnetic heavy fermion system $\mathrm{U}_{4} \mathrm{Ru}_{7} \mathrm{Ge}_{6}$ as it is driven to the paramagnetic state under applied pressure. The results of the transport properties under pressure show no sign of a discontinuous behavior as $T_{C}$ is reduced. The resistivity curves present no hysteresis effects for any pressure, before and after ferromagnetism is suppressed. Disorder is certainly present in our system, as evidenced by its high residual resistivity. It is possible that the continuous behavior is due to its influence since it substantially modifies the properties of our sample when compared to 
single crystals [11]. However, disorder is not sufficiently strong to give rise to localization effects or Griffith's singularities. On the contrary, our sample presents many of the properties expected for an itinerant clean system, as the $T^{5 / 3}$ temperature dependence of the resistivity at its FQCP.

$\mathrm{U}_{4} \mathrm{Ru}_{7} \mathrm{Ge}_{6}$ is a unique Uranium compound with negligible anisotropy. This implies that spin-waves, the elementary excitations of a ferromagnetic metal can be easily excited and play a fundamental role in the thermodynamic and transport properties of this system at low temperatures. We have shown that as the FQCP is approached with increasing pressure the spin-wave stiffness softens and we obtain its quantum critical behavior. It would be very interesting to measure directly the spinwave stiffness of $\mathrm{U}_{4} \mathrm{Ru}_{7} \mathrm{Ge}_{6}$ by inelastic neutron scattering and compare it to the results we obtained. Specially interesting would be to observe with neutrons the softening of the magnons with increasing pressure.

Although our results are in close agreement with those of Refs. [5] and [10] on the same system, they differ from those obtained in single crystals [11] where a reorienta- tion of the magnetization inside the ferromagnetic phase is observed.

In summary our results on the ferromagnetic $\mathrm{U}_{4} \mathrm{Ru}_{7} \mathrm{Ge}_{6}$ provide strong evidence for the existence of a pressure induced ferro-para quantum phase transition in this system that is accompanied by a softening of the elementary excitations of the ordered phase. Its quantum critical behavior shares many features with that expected for a clean, itinerant FQCP.

\section{ACKNOWLEDGMENTS}

The authors M.P.N., M.A.C., A.L., A.L., D.C.F., J.L.J., C.E., J.F.O., E.B.S. and M.B.F. would like to thank the Brazilian Agencies CAPES, CNPq and FAPERJ for partial financial support. We thank Prof. Dr. Renato Bastos Guimarães for using the X-Ray Diffraction Laboratory (LDRX) at the Instituto de Física, Universidade Federal Fluminense (IF-UFF).
[1] see Strongly correlated electron systems; reports on the progress of the field, Guest Editors, Laura H Greene, Joe Thompson and Jrg Schmalian, Reports on Progress in Physics, 80, Number 3 (2017); Ye Chen, Zongfa Weng, Smidman Michael, Xin Lu and Huiqiu Yuan, Chinese Phys. B 25077401 (2016).

[2] Dai Aoki and Jacques Flouquet, J. Phys. Soc. of Jap. 81 011003 (2012).

[3] Mucio A. Continentino, Quantum scaling in many-body systems: an approach to quantum phase transitions, Cambridge University Press (2017).

[4] L. G. Akselrud, J. P. Jarmoljuk, and E. I. Gladyshevskij, Dopov Akad Nauk., 359 (1978).

[5] S. A. M. Mentink, G. J. Nieuwenhuys, A. A. Menovsky, and J. A. Mydosh, J. Appl. Phys. 695484 (1991).

[6] H. H. Hill, Plutonium 1970 and Other Actinides, edited by W. N. Miner (American Institute of Mining, Metallurgical, and Petroleum Engineers, New York, 1970), p. 2.

[7] B. Lloret, B. Buffat, B. Chevalier, and J. Etourneau, J. Mag. Mag. Mat. 67232 (1987).

[8] E. Colineau, F. Wastin, E. J. Higgins, and J. Rebizant, J. Alloy. Comp. 317, 336 (2001).

[9] Samir F. Matar, Bernard Chevalier, and Rainer Pöttgen, Solid State Sci. 275 (2014).

[10] Hiroyuki Hidaka, Sosuke Takahashi, Yusei Shimizu, Tatsuya Yanagisawa, and Hiroshi Amitsuka, J. Phys. Soc. Jpn. 80 SA102 (2011).

[11] Michal Vališka, Martin Diviš, and Vladimír Sechovsky, Phys. Rev. B 95085142 (2017); Michal Vališka, Milan Klicpera, Petr Doležal, Oscar Fabelo, Anne Stunault, Martin Diviš, Vladimír Sechovský, arXiv:1711.06113 and Phys. Rev. B 97, 125128 (2018).

[12] Frederic Keffer, in Encyclopedia of Physics, SpringerVerlag, Vol. XVIII/2 pp. 1-273 (1966).
[13] M. Pajda, J. Kudrnovsky, I. Turek,V. Drchal, and P. Bruno, Phys. Rev. B64 174402 (2001).

[14] J. Mathon, Proc. Roy. Soc. A 306355 (1968).

[15] J. Larrea J., M. B. Fontes, A. D. Alvarenga, E. M. Baggio-Saitovitch, T. Burghardt, A. Eichler, and M. A. Continentino, Phys. Rev. B 72, 035129 (2005).

[16] Mucio A. Continentino, Gloria M. Japiassu, Amos Troper, Phys. Rev. 39, 9734 (1989).

[17] H. M. Rietveld, J. Appl. Cryst. 2, 65 (1969).

[18] J. Rodríguez-Carvajal, Physica B 192, 55 (1993); T. Roisnel and J. Rodríguez-Carvajal, Materials Science Forum 378?381, 118 (2001)

[19] Institut Laue-Langevin, http://www.ill.eu/sites/fullprof/Php/d

[20] George F. Vander Voort, Metallography - Principles and Practice, ASM International, Ohio, 2007.

[21] D. J. Poutcharovsky and E. Parthe, Acta Cryst. B30, 2692 (1974).

[22] Ingmar Grenthe, Janusz Drozdzynski, Takeo Fujino, Edgar C. Buck, Thomas E. Albrecht-Schmitt, and Stephen F. Wolf, Uranium in: The Chemistry of the Actinide and Transactinide Elements, volume 1, 3rd edition - Edited by: Lester R. Morss, Norman M. Edelstein and Jean Fuger, Springer, Dordrecht, 2008.

[23] Nathan R. Gubel. Kenneth H. Eckelmeyer. Kimbberly N. Johnson. Mark J. Jacksone Jonatan S. Morrell, Introduction to Uranium in: Uranium Processing and Properties - Edited by: Jonathan S. Morrell e Mark J. Jackson, Springer, New York, 2013.

[24] C. P. Susz, J. Muller, K. Yvon, and E. Parth, J. Less Common Met. 71, 1 (1980).

[25] Sigurds Arajs, Robert H. Flora, and Elmer E. Anderson, J. Nucl. Mater. 37, 89 (1970).

[26] L. F. Bates and D. Hughes, Proc. Phys. Soc. B67, 28 (1954). 
[27] C. J. Kriessman Jr and T. R. McGuire, Phys. Rev. 85, 71 (1952).

[28] C. P. Susz, J. Muller, K. Yvon, and E. Parth, J. Less Common Met. 71, 1 (1980).

[29] D. J. Poutcharovsky, K. Yvon, and E. Parthe, J. Less Common Met. 40, 139 (1975).

[30] see Stephen Blundell in Magnetism in condensed matter, Oxford University Press, p.195-196, (2001).

[31] Frederic Keffer, Spin Waves in: Encyclopedia of Physics: Ferromagnetism. Edited by: H. P. J. Wijn, Chief Editor: S. Flügge, Springer, Berlin, (1966).

[32] T. M. Palstra, A. A. Menovsky, J. Van den Berg, A. J. Dirkmaat, P. H. Kes, G. J. Nieuwenhuys, and J. A. Mydosh. Phys. Rev. Lett. 55, 2727 (1985).

[33] W. Schlabitz, J. Baumann, B. Pollit, U. Rauchschwalbe, H. M. Mayer, U. Ahlheim, and C. D. Bredl, Superconductivity and magnetic order in a strongly interacting fermi-system: $\mathrm{URu}_{2} \mathrm{Si}_{2}$ In: Ten Years of Superconductivity: 1980-1990, pages 89-95, Springer, 1986.

[34] S. K. Dhar, R. J. Begum, P. Raj, P. Suryanarayana, L. C. Gupta, and R. Vijaya-raghavan, Solid State Commun. 83, 965 (1992).

[35] T. Mito, M. Hattori, G. Motoyama, Y. Sakai, T. Koyama, K. Ueda, T. Kohara,M. Yokoyama, and H. Amitsuka, J. Phys. Conf. Ser. 391, 012044 (2012).
[36] B. Lloret, B. Chevalier, P. Gravereau, B. Darriet, and J. Etourneau, J. Phys. Colloq. 49, C8-487 (1988).

[37] R. Troc and V. H. Tran, , J. Mag. Mag. Mat. 73, 389 (1988).

[38] A. Vernière, P. Lejay, P. Bordet, J. Chenavas, J. P. Brison, P. Haen, and J. X. Boucherle, J. Alloys Compd. 209, 251 (1994).

[39] D. Belitz, T. R. Kirkpatrick, A. J. Millis and Thomas Vojta, Phys. Rev. B58, 14155 (1998).

[40] G. R. Stewart, Rev. Mod. Phys. 56, 755 (1984).

[41] I. Sheikin, Y. Wang, F. Bouquet, P. Lejay and A. Junod, J. Phys.: Condens. Matter 14, L543 (2002).

[42] N. H. Andersen and H. Smith, Phys. Rev. B 19 (1979), 384. M. B. Fontes, J. C. Trochez, B. Giordanengo, S. L. Budko, D. R. Sanchez, and E. M. Baggio-Saitovitch and M. A. Continentino, Phys. Rev. B60, 6781 (1999).

[43] B. Raquet, M. Viret, E. Sondergard, O. Cespedes, and R. Mamy, Phys. Rev. B66, 024433 (2002) and references therein.

[44] M. Brando, D. Belitz, F. M. Grosche, and T. R. Kirkpatrick, Rev. Mod. Phys. 88, 25006 (2016).

[45] Alexander Steppke, Robert Kchler, Stefan Lausberg, Edit Lengyel, Lucia Steinke, Robert Borth, Thomas Lhmann, Cornelius Krellner, Michael Nicklas, Christoph Geibel, Frank Steglich, Manuel Brando, Science 339, 933 (2013). 